\title{
mir-21a-5p promote the progress of inflammation after Traumatic Spinal Cord Injury via up-regulating neurotoxic reactive astrocyte (A1) polarization by inhibiting CNTF/STAT3/Nkrf pathway
}

\author{
Yining Zhang \\ shandong first medical university \\ Tingting Meng \\ shandong university \\ Jianan Chen \\ shandong university \\ Ying Zhang \\ shandong university \\ Jianning Kang \\ shandong first medical university

\section{Xinyu Li} \\ shandong university \\ Guilian Yu \\ shandong university \\ Lige Tian \\ shandong university

\section{Zhengxin Jin} \\ shandong first medical university \\ Hui Dong \\ shandong university
}

BIN NING ( $\nabla$ ningbin@sdu.edu.cn)

Jinan Central Hospital Affiliated to Shandong University https://orcid.org/0000-0002-7592-9485

\section{Research}

Keywords: microRNA-21a-5p, Traumatic Spinal Cord Injury (TSCI), neurotoxic reactive astrocyte (A1s), ciliary neurotrophic factor (CNTF), ciliary neurotrophic factor receptor a (CNTFR a)

Posted Date: December 28th, 2020 
DOI: https://doi.org/10.21203/rs.3.rs-135108/v1

License: (c) (1) This work is licensed under a Creative Commons Attribution 4.0 International License. Read Full License 


\section{Abstract \\ Background}

Reactive astrocytes play an important role in Traumatic Spinal Cord Injury (TSCI). Interestingly, naive astrocytes can easily transform into neurotoxic reactive astrocytes(A1s) when inflammatory stimulation occurs. Previous researches have reported that miR-21a-5p is involved in the regulation of various stages of Spinal Cord Injury (SCI). However, it is not clear whether miR-21a-5p affected the polarization of reactive astrocytes. The purpose of our study was to detect the effects and mechanism of miR-21a-5p in the induction of neurotoxic reactive astrocytes (A1s) formation.

\section{Methods}

Gene chip assay and qRT-PCR were used to detect the expression of Cntfr $a$ in TSCI models or sham operation. Bioinformatics analysis was used to speculate the potential targeting of miR-21a-5p, which was further confirmed by qRT-PCR, western blotting, a dual-luciferase reporter assay, and RNA pulldown assay. In vivo, the TSCI model was performed by a $68099 \otimes$ precision percussion device, and the A1s phenotype was identified by immunofluorescence staining. In vitro, A1s were induced by IL-1 a, TNF-a, and C1q. A1s and neuroprotective reactive astrocytes (A2s) markers were confirmed by qRT-PCR, western blotting, and immunofluorescence. ChIP assay was used to explore the targeting gene of STAT3, the downstream of Cntfr a.

\section{Results}

The expression of miR-21a-5p was significantly increased while Cntfr a was decreased since naive astrocytes transformed into A1s after 3 days post-TSCI. In addition, the mRNA and protein of Cntfr a were decreased while miR-21a-5p was overexpressed. The binding site between miR-21a-5p and Cntfr a was further confirmed by the dual-luciferase reporter and RNA pulldown assay. We also discovered that A1s markers were decreased while markers of A2s were increased with the pretreatment of CNTF. Chromatin immunoprecipitation (ChIP) assay was used to prove that CNTF inhibited A1s induction by activating the expression of Nkrf via the CNTF/STAT3 pathway. Downregulation of miR-21a-5p enhanced the inhibitory effect of CNTF in A1s in vitro. In vivo, the expression of A1s markers significantly decreased with the treatment of antagomir-21, while Cntfr a siRNA treatment was just the opposite.

\section{Conclusion}

We observed that increased miR-21a-5p down-regulated Cntfr a in A1s induced by $\mathrm{TSCl}$, promoting the inflammatory process. In addition, we also identified the effect and potential mechanism of CNTF, a 
specific ligand of CNTFR $a$, on inhibiting naive astrocytes transformed into A1s for the first time. Collectively, our studies demonstrated that targeting miR-21a-5p is a prospective therapy for curing TSCl.

\section{Background}

Traumatic Spinal Cord Injury (TSCl), the most serious damage after spinal traumatic injury, usually leads to lifelong disability due to the destroyed continuity of the spinal cord [1-3]. Previous studies have been reported that the cost of life-long treatment per patient is at least \$1.1 million in the United States $[4,5]$. However, there is still no effective treatment for TSCl at present. However, there is still no effective treatment for $\mathrm{TSCl}$ at present. Amanda Phuong Tran et.al reported that neuroinflammation was associated with the weakening axonal regenerative activity, leading to the poor recovery of injured tissue in the early stage of $\mathrm{TSCl}$, which might influence the prognosis of TSCI [6].

Astrocytes are the most abundant resident cells in Central Nervous System (CNS), exerting a housekeeping role in healthy $\mathrm{CNS}[7,8]$. However, native astrocytes trended to be induced into reactive astrocytes when CNS diseases occurred [9]. Besides neuroprotective effects[10], reactive astrocytes also regulated neuroinflammation response to CNS damage[8, 11-13]. Recently, serious studies have found that reactive astrocytes can be divided into two kinds of phenotypes: neurotoxic reactive astrocytes(A1s) and neuroprotective reactive astrocytes(A2s). A1s were induced by IL-1a, TNF- $\mathrm{a}$ and $\mathrm{C} 1 \mathrm{q}$ secreted by M1 microglia (LPS-stimulated) [14-17] while A2s were induced by ischemia[14, 15, 18]. Just like M1 microglia/macrophage, A1s lost the original function of astrocytes but increased the secretion of inflammatory cytokines $[12,14,16,19]$. On the other hand, A2s up-regulated neurotrophic factors and promoted the survival of neurons[18, 20,21]. Marks of A1s includes complement component 3 (C3), serpin family G member 1(Serping1) and histocompatibility 2, D region locus 1(H2D1). S100 calciumbinding protein $\mathrm{A} 10(\mathrm{~S} 100 \mathrm{a} 10)[14,16]$ is the marker of $\mathrm{A} 2 \mathrm{~s}$.

Ciliary neurotrophic factor receptor a(CNTFR a), a receptor of ciliary neurotrophic factor(CNTF) [22, 23], is widely expressed in CNS[23, 24]. Through combining with CNTF, CNTFR a activates the JAK/STAT3 signal pathway[22, 23]. At first, CNTF was found to be required for axonal regeneration after Spinal Cord Injury (SCI)[23, 25-27]. Subsequently, CNTFR a has been found to exert a role of neuroprotection and relieve neuroinflammation by regulating astrogliosis[23, 28-30] and the function of microglia[31-34]. Previous researches reported that NF-KB signal pathway has been proved to be essential for A1s polarization $[15,35,36]$ while STAT3 signal pathway might promote A2s and inhibit A1s transformation $[15,18]$. Similarly, STAT3 also might be essential for glial scar formation and astrocytic neuroprotection after SCI[10, 30, 37-39]. It is worth mentioning whether CNTF affects the polarization of reactive astrocytes through STAT3 signal pathway has not been reported.

MicroRNA, a kind of small non-coding RNA containing 19-25 nucleotides[40], can down-regulate the expression of mRNA via targeting the 3'UTR[40, 41]. Recently, several studies have reported that microRNA might be necessary for the recovery of SCI[42, 43]. Significantly, miR-21a-5p regulates the formation of glial scars[44] and fibrosis[45, 46], inhibiting the axon regeneration after SCI[47] as we 
previous described. In addition, miR-21a-5p was also confirmed to promote the polarization of A2s[18] in Ischemic Spinal Cord Injury (ISCI). Nevertheless, it is still unclear whether miR-21a-5p exerted a key role in regulating the induction of $\mathrm{A} 1 \mathrm{~s}$ after $\mathrm{TSCl}$.

In this study, we aim to explore the relationship between miR-21a-5p and the polarization of reactive astrocytes, and detect possible mechanism in TSCl. Our study provides a prospective viewpoint of the polarization of reactive astrocytes, offering possible solutions for TSCl repairment.

\section{Materials And Methods}

\section{Ethics statement}

All operations are performed according to the animal care standards of the Chinese National Institute of Health and regulations of Shandong First Medical University Committee for the Care and Use of Laboratory Animals.

\section{Animals and TSCl model}

Male C57BL/6 background mice (6-8 weeks) were purchased from the Pengyue Experimental Animal breeding Co., Ltd. (Jinan, China). The animals were housed in a clean cage under conditions of $22-24^{\circ} \mathrm{C}$, relative humidity of $30-60 \%$, and a 12 -h light/dark cycle, with adequate food and water.

Mice were anesthetized with $3 \%$ pentobarbital (30 mg/kg, i.p.), then T8-T10 laminectomy was performed to expose the spinal cord. TSCI model was completed by $68099 \rrbracket$ precision percussion (RWD Life Science, Shenzhen, China; $1 \mathrm{~m} / \mathrm{s}$ speed, $2 \mathrm{~mm}$ depth, 1 second dwell time). The success of the model was confirmed by tail spasms and retraction-like flutters of the legs.

\section{Animal experiments}

a. Mice were divided into sham and TSCl. The sham and 3 days post-TSCI mice spinal cord tissues were used for gene chip assay to detect the differential expression of mRNA.

b. Mice were randomly divided into 4 groups: sham, negative control (NC), antagomir-21, and antagomir$21+\mathrm{cntfr}$ siRNA. In NC or antagomir-21 groups, antagomir-NC or antagomir-21 $(2.5 \mu \mathrm{l}, 2 \mu \mathrm{mol} / \mathrm{ml})$ (RiboBio, Guangzhou, China) were intrathecally injected for 3 days $(0,1,2)$ after mice were performed to $\mathrm{TSCl}$, and siRNA NC ( $1 \mu \mathrm{l}, 0.5 \mu \mathrm{mol} / \mathrm{ml}$, RiboBio) were intrathecally injected for 1 day $(0)$. In antagomir-21+cntfr siRNA group, after treated intrathecally with antagomir-21, Cntfr siRNA $0.5 \mathrm{nmol}(1 \mu \mathrm{l}, 0.5 \mu \mathrm{mol} / \mathrm{ml})$ were intrathecally injected for 1 day (0). In the sham group, only performed laminectomy.

3 days after $\mathrm{TSCl}$, mice were anesthetized with $3 \%$ pentobarbital, then spinal cord tissues were carefully removed after transcardial perfusion. For staining use, the tissues were fixed overnight with $4 \%$ paraformaldehyde at $4^{\circ} \mathrm{C}$. Then, after dehydrated in xylene and gradient alcohol solutions, the tissues 
were embedded in paraffin. For gene chip analysis, qRT-PCR, and western blotting use, the sample was quickly placed in a frozen tube and put in liquid nitrogen, or directly extracted total RNA or protein.

\section{mRNA gene chip assays}

The tissues were extracted from sham and 3 days post-TSCI mice and frozen in liquid nitrogen. Gene chip assays were performed by Genechem Co. (Shanghai, China).

\section{Bioinformatics analysis}

PicTar (https://pictar.mdc-berlin.de/), TargetScan (http://www.targetscan.org/mmu_72/), miRanDa (http://www.microrna.org/microrna/getDownloads.do) bioinformatics were used to predict potential target genes of miR-21a-5p.

\section{Cell culture and transfection}

Cells were cultured in Dulbecco's modified essential medium (Gibco, China) with $10 \%$ fetal bovine serum (Gibco, Australia), and 1\% penicillin-streptomycin (Solarbio, China), and were placed in a humidified incubator with $5 \% \mathrm{CO} 2$ at $37^{\circ} \mathrm{C}$.

Primary mouse astrocytes were extracted from 1-3 days C57BL/6, the brain and spinal cord were dissected layer by layer; the brain and spinal cord were separated; removed the meninges and blood vessels, and the brain and spinal cord were extracted. After being fragmented, tissues were digested with trypsin (Solarbio) at $37{ }^{\circ} \mathrm{C}$ for 15 minutes. And the cell suspension was centrifuged and re-suspended in the cell culture solution. An 1h pre-plating step was performed to further remove fibroblast. The unattached astrocytes were planted in a $100 \mathrm{~mm}$ dish. The $3 \mathrm{rd}$-generation astrocytes were used for researches.

For transfection, miR-21 mimic, inhibitor or negative control, and (or) Cntfr siRNA or siRNA NC (RiboBio, Wuhan, China) were transfected into astrocytes when they were $50 \%-70 \%$ density. The transfection was performed with the help of opti-MEM (gibco) and Lipofectamine ${ }^{\circledR 2} 2000$ Reagent (Invitrogen, ThermoFisher, Shanghai, China). And then, after pretreatment with CNTF (100ng/ml, PeproTech) for 24h, cells were treated with IL-1a (3ng/ml, PeproTech), TNF-a (30ng/ml, PeproTech) and C1q (400ng/ml, CUSABIO) for inducing into A1s. RNA or protein was extracted for subsequently use.

\section{RNA extraction and quantitative real-time polymerase chain reaction (qRT-PCR)}

TRIzol $^{\mathrm{TM}}$ (Accurate Biology, Hunan, China) was used to extract total RNA from astrocytes or spinal cord tissue. The SpectraMax®QuickDrop ${ }^{\mathrm{TM}}$ spectrophotometer was used for detecting the concentration of total RNA. The total RNA was stored at $-80^{\circ} \mathrm{C}$ or directly used.

Mir- $\mathrm{X}^{\mathrm{TM}}$ miRNA First-Strand Synthesis Kit (Takara, Dalian, China) and miR-21a-5p primer (Takara) were used to detect the expression level of miR-21a-5p, and U6 was used as an endogenous control to 
normalize the results. The 1000ng total RNA was prepared for reverse transcription using Exo M-MLV RT Kit with gDNA Clean for qPCR II (Accurate Biology). We performed qRT-PCR with the SYBR®Green Premix Pro Taq HS qPCR Kit (Accurate Biology) at LightCycler®480II Fast Real-Time PCR System (Roche, Switzerland). GAPDH was used as an endogenous control to normalize the results. The results were calculated with the value $2^{-\Delta \Delta C T}$. The primer pairs used are in Table. 1.

\section{Protein extraction and western blotting}

Astrocytes or spinal cord tissue were lysed by RIPA (Solarbio) with 1\% PMSF (Beyotime Biotechnology, Shanghai, China) and 1\% Phosphatase Inhibitor Cocktail 100X (CWBio, Beijing, China). The concentration of protein was measured by BCA detection kit (Beyotime Biotechnology). The protein was stored at $-80{ }^{\circ} \mathrm{C}$ or directly used.

The protein samples were separated in SDS-PAGE gel (Beyotime Biotechnology) and transferred to polyvinylidene difluoride membranes (Millipore, USA). After blocking in 5\% Skim milk powder (BioFroxx, Germany) at room temperature for 1 hour, membranes were incubated with primary antibodies at $4^{\circ} \mathrm{C}$ overnight. The next day, after washing with PBST, membranes were incubated with secondary antibodies at room temperature for $1 \mathrm{~h}$. Finally, images were detected by FluorChem M (ProteinSimple, USA).

\section{Antibodies used were as follows:}

CNTFR a (1:1000, Santa Cruz, USA), Phospho-STAT3 (p-Stat3, 1:2000, Cell Signal Technology, USA), STAT3 (1:1000, Cell Signal Technology), iNOS (1:1000, Cell Signal Technology), Complement component 3 (C3, 1:50, Abcam, UK), S100a10 (1:1000, Abcam), $\beta$-actin (1:5000, ZSGB-Bio, Beijing, China), goat antirabbit secondary antibody (1:5000, ZSGB-Bio), goat anti-mouse secondary antibody (1:5000, ZSGB-Bio), goat anti-rat secondary antibody (1:5000, ZSGB-Bio).

\section{Enzyme-linked immunosorbent assay (ELISA)}

The concentration of IL-1 $\beta$ released by A1s was detected by Enzyme-linked immunosorbent assay (ELISA) for quantitative detection of mouse IL-1 $\beta$ Kit (Invitrogen, ThermoFisher Scientific). The experimental operation was performed according to the protocol of the kit. The colorimetric optical density (OD) was measured by SpectraMax®i3x enzyme labeling instrument (Molecular Devices, USA).

\section{Dual-luciferase reporter assay}

HEK 293t cells were planted in a 24-well plate. For the construction of plasmids, WT or MUT Cntfr a 3'UTR fragments were inserted into PmirGLO Dual-Luciferase miRNA Target Expression Vector (BioSune, Jinan, China). Then, plasmids, miR-21a-5p mimic, inhibitor, and negative control were transfected into HEK 293t cells. After 48 hours, added $1 \times$ PLA cell Lysis Buffer and shaken to lyse the cells at room temperature for $15 \mathrm{~min}$, then collected the cell lysate. $20 \mu \mathrm{l}$ cell lysate was added to a dedicated 96 -well plate, then $100 \mu \mathrm{l}$

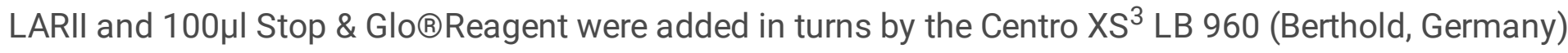
and MikroWin software to detect the firefly luciferase activities and Renilla luciferase activities 
respectively. We completed this experiment using the Promega Dual-Luciferase system (Promega, Madison, USA). The difference of luciferase activity between firefly and Renilla was calculated and analyzed.

\section{RNA pull-down assay}

Mus-Cntfr in pcDNA3.1 (+), antisense-MUT-Cntfr in pcDNA3.1 (+), and pcDNA3.1 (+) were linearized with restriction enzymes, then used for in vitro transcription. In in vitro transcription, we used the MEGAscript T7 Kit (Ambion, Thermo Fisher Scientific, Shanghai, China) and biotin 16 UTP (Ambion, Thermo Fisher Scientific, Shanghai, China) for biotin-labeled RNA transcripts. MEGA clear Kits (Ambion, Thermo Fisher Scientific, Shanghai, China) were used for purification in vitro. $3 \mu \mathrm{g}$ of biotinylated RNA was heated at 90 ${ }^{\circ} \mathrm{C}$ for $5 \mathrm{~min}$ and then laid at room temperature for $30 \mathrm{~min}$, then cooled to $4{ }^{\circ} \mathrm{C}$. RNA was mixed with $1 \mathrm{mg}$ protein extracted from astrocytes, incubated with shaking at room temperature for $3 \mathrm{~h}$. Each binding

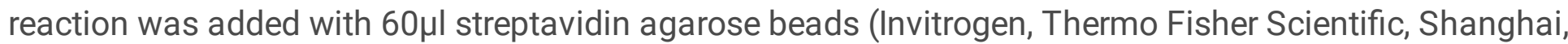
China) and incubated with a rolling shaker at room temperature for $2 \mathrm{~h}$. Then, a qRT-PCR assay was used to detect the expression of miR-21a-5p in pull-down RNA.

\section{Immunofluorescence}

For astrocytes, the cells planted in a 24-well plate were washed with PBS 3 times, treated with 4\% paraformaldehyde (Solarbio) for $15 \mathrm{~min}, 0.5 \%$ Triton X-100 penetrated for $10 \mathrm{~min}$, and then blocked with $10 \%$ normal goat serum (Solarbio) for 1 hour. Then, cells were incubated with primary antibody at $4{ }^{\circ} \mathrm{C}$ overnight. The next day, samples were incubated with secondary antibodies for $30 \mathrm{~min}$ at room temperature. Finally, Antifade Mounting Medium with DAPI (Beyotime Biotechnology) was used for sealing.

Spinal cord tissues were sectioned after paraffin embedding. After treated with Environmentally friendly transparent dewaxing liquid (Solarbio) for deparaffinizing, slices were hydrated in $100 \%, 95 \%, 90 \%, 80 \%$, $70 \%$ ethanol solution in turns. Then, sections were heated in citrate buffer for $15 \mathrm{~min}$ for antigen retrieval. The procedure of staining was the same as that was performed in cells.

An upright fluorescence microscope (Olympus, Tokyo, Japan) was used for obtaining images.

\section{Antibodies used were as follows:}

Mouse anti-GFAP (1:200, Cell Signal Technology, USA), Rat anti-C3 (1:20, Abcam, UK), Rabbit antiS100a10 (1:200, Abcam), Alexa Fluor ${ }^{\circledR} 488$ goat anti-mouse IgG (1:200, Abcam), Alexa Fluor ${ }^{\circledR 594}$ goat anti-rat IgG (1:200, Abcam), Alexa Fluor ${ }^{\circledR} 594$ goat anti-rabbit IgG (1:200, Abcam).

\section{Immunohistochemistry}

After antigen retrieval (see in Immunofluorescence), spinal cord tissues were incubated in $3 \% \mathrm{H}_{2} \mathrm{O}_{2}$. Then, sections were blocked with 3\%BSA (BioFroxx) at room temperature for 1 hour. The slices were incubated 
with rat anti-C3 (1:20, Abcam, UK) primary antibodies at $4{ }^{\circ} \mathrm{C}$ overnight. The next day, after washed with PBS 3 times, goat anti-rat secondary antibody (1:200, ZSGB-Bio) was used for incubating at room temperature for 30 minutes and stained with 3,3'-diaminobenzidine tetrahydrochloride (DAB, ZSGB-Bio). Next, sections were stained with hematoxylin (Solarbio) and differentiated with alcohol-HCl. After rinsing with tap water, slices were dehydrated in $70 \%, 80 \%, 90 \%, 95 \%$, and $100 \%$ ethanol solution in turns, and made transparent with Environmentally friendly transparent dewaxing liquid (Solarbio). Then sealed with neutral gum and dried. Images were obtained with an upright fluorescence microscope (Olympus, Tokyo, Japan).

\section{Chromatin immunoprecipitation (ChIP)}

ChIP assay was performed according to the manufacturer's protocols in Chromatin Extraction Kit (Abcam) and ChIP Kit Magnetic-One Step (Abcam). The chromatin was extracted from astrocytes and sonicated into 200-1000bp fragments. Then, the chromatin was immunoprecipitated with the anti-p-Stat3 antibody. The primer sequences of Nkrf promoter were as follows: Forward: 5'-

AACCCCTTTCCAAGGACACAG-3'; Reverse: 5'-AGACTCCTGGTAGGGGACTC-3'. The precipitated chromatin was used for qRT-PCR or PCR. The 100 150 bps PCR products were electrophoresed through a $2 \%$ agarose gel (baygene, Beijing, China) with GelRed ${ }^{\text {TM }}$ Nucleic Acid Gel Staining solution (biosharp, China), and visualized in UV illumination with FluorChem M (ProteinSimple, USA).

\section{Statistical analysis}

GraphPad Prism v8.0 software (La Jolla, CA, USA) and SPSS v22.0 software (IBM, Chicago, IL, USA) was used for statistical analysis. Differences between two groups were analyzed by Student's t-test. Data were presented as mean \pm standard deviation (SD). One-way analysis of variance (ANOVA) with Bonferroni's tests was performed for comparisons between multiple groups. $\mathrm{P}<0.05$ showed statistical significance.

\section{Results}

\section{The expression of miRNA and mRNA in A1 reactive astrocytes induced by TSCl}

To determine the expression of miR-21a-5p after TSCl, we performed a qRT-PCR analysis between the sham operation and 3-days post-TSCI. The expression of miR-21a-5p after TSCI was significantly increased compared with the sham group (Fig. 1A). Immunofluorescence staining showed the increased expression of $\mathrm{C} 3$, an $\mathrm{A} 1 \mathrm{~s}$ marker, in $\mathrm{GFAP}^{+}$cells after TSCl (Fig. 1B), indicating the polarization of $\mathrm{A} 1 \mathrm{~s}$. To verify the effect of IL-1a $(3 \mathrm{ng} / \mathrm{ml})$, TNF-a $(30 \mathrm{ng} / \mathrm{ml})$ and C1q $(400 \mathrm{ng} / \mathrm{ml})$ on inducing A1s, qRT-PCR was used to detect the expression of mRNA. As shown in Fig. 1C, the expression of C3, Serping1, H2d1 were up-regulated and S100a10 was down-regulated in A1 model group. Meanwhile, the expression of miR21a-5p was also up-regulated in A1s (Fig. 1D), which indicated that miR-21a-5p may affect the induction of $\mathrm{A} 1 \mathrm{~s}$. 
To verify the targeting gene of miR-21a-5p, a gene chip assay was performed between the 3-d post-TSCI and sham operation groups. Furthermore, bioinformatics analysis indicated that Cntfr a, Epha4, Pitx2, or Abcd2 might exist a binding sequence of miR-21a-5p (Fig. 1E). qRT-PCR further demonstrated the mRNAs level of Cntfr a, Epha4, Pitx2 were all decreased in 3-days post-TSCl group (Fig. 1F, Supplementary Fig. S1A-C), suggesting the possibility of them being the targeting gene of miR-21a-5p.

\section{MiR-21a-5p decreased the expression of Cntfr a by targeting $3^{\prime} U T R$ in astrocytes}

In order to further confirm the target of miR-21a-5p, astrocytes were treated with miR-21a-5p mimic, inhibitor, or negative control. And then, qRT-PCR was used to determine the mRNA expression in astrocytes. Results showed that miR-21a-5p overexpression significantly decreased the expression of Cntfr a and Epha4 (Fig. 2A, Supplementary Fig. S2A) but did not affect Pitx2 (Supplementary Fig. S2B). Meanwhile, the level of Cntfr $a$ and Epha4 were significantly increased after down-regulating miR-21a-5p (Fig. 2B, Supplementary Fig. S2C). Moreover, western blotting also showed similar results on CNTFR a protein expression (Fig. 2C). Here, we confirmed that miR-21a-5p might regulate the polarization of A1s through targeting Cntfr a, as the expression of Cntfr a was markedly decreased in A1s reactive astrocytes (Supplementary Fig. S2D), which was different form Epha4 (Supplementary Fig. S2E).

Dual-luciferase reporter assay was performed to verify the binding of miR-21a-5p and Cntfr $a$. The luciferase activity of Cntfr a-WT was decreased in the miR-21a-5p mimic group but increased in the miR$21 a-5 p$ inhibitor group. However, there was no statistical difference in luciferase activities of Cntfr a-MUT between miR-21a-5p mimic and inhibitor groups (Fig. 2D). And then, RNA pulldown assay was used to further verify the binding condition of Cntfr a to miR-21a-5p in astrocytes (Fig. 2E). To sum up, miR-21a$5 p$ decreased the Cntfr a expression by targeting 3'UTR.

Subsequently, to verify that miR-21a-5p regulates inflammation through Cntfr a, miR-21a-5p inhibitor was transfected into A1s. The results showed that the expression of Cntfr a was enhanced in miR-21a-5p inhibitor group versus the control group in A1s (Fig. 2F).

CNTFR is a specific receptor of ciliary neurotrophic factor (CNTF), which can activate the STAT3 signal pathway. To confirm the influence of miR-21a-5p on the activation of CNTF/STAT3 pathway, miR-21a-5p mimic and inhibitor were transfected into astrocytes. Western blotting showed that the phosphorylation of STAT3 was enhanced in the miR-21a-5p overexpression group (Fig. 2G) but weakened in a miR-21a-5p knockdown group (Fig. 2H).

Our results suggested that miR-21a-5p weakened the function of CNTF through targeting Cntfr a 3'UTR.

\section{CNTF inhibited the induction of A1s via promoting STAT3/Nkrf pathway}

To confirm the effect of CNTF/CNTFR a on the polarization of relative astrocytes, astrocytes were pretreated with CNTF for 24 hours. The results of qRT-PCR, western blotting, and ELISA assay showed that 
CNTF significantly decreased iNOS and IL-1 $\beta$ expression in A1s (Fig. 3A, G, H). Moreover, CNTF reduced C3, Serping1, H2d1 expression but up-regulated S100a10 expression in A1s (Fig. 3C-F). Western blotting also showed the same results in $\mathrm{C} 3$ and S100a10 protein levels (Fig. 3H). Meanwhile,

immunofluorescence staining showed high C3 expression and low S100a10 expression in $\mathrm{GFAP}^{+}$cells in A1s, which reversed by CNTF (Fig. 31-J). Furthermore, native astrocytes treated with CNTF exhibited slightly low of A1s markers expression and high A2s marker expression. Those data confirmed that CNTF could exert an anti-inflammatory effect by inhibiting the induction of A1s.

Since CNTF activates STAT3 signal pathway, S3I-201 $(10 \mu \mathrm{M})$ was used to repress the activation of STAT3 pathway (DMSO used as a control group). As shown in Fig. 4A-D, S3I-201 treatment significantly increased C3, Serping1, and H2d1 expression but decreased S100a10. Those results suggested that CNTF inhibited the induction of A1s through the STAT3 signal pathway. But how transcription factor STAT3 plays a role in A1s induction was still unclear.

Known that the NF-kB signaling pathway plays an important role in the induction of A1s and NF-KB repressing factor (Nkrf) can effectively inhibit the NF-kB pathway, we speculated that Nkrf might affect the induction of $\mathrm{A} 1 \mathrm{~s}$. As shown in Fig. 4E, pre-treating with CNTF significantly reserved the low Nkrf expression in A1s, and this effect was restrained in the S3I-201 treatment group. And then, we detected whether Nkrf expression could be promoted by the transcription factor STAT3. ChIP assay further confirmed that the Nkrf promoter was significantly enriched in p-STAT3, which demonstrated the important role of STAT3 in Nkrf expression (Fig. 4F).

In conclusion, these data strongly confirmed that CNTF inhibits the induction of A1s through STAT3/Nkrf pathway.

\section{MiR-21a-5p promoted the induction of A1s by suppressing the effect of CNTF}

To verify whether miR-21a-5p affected the function of CNTF, miR-21 mimic and inhibitor were transfected into astrocytes, and then pre-treated with CNTF. The inhibition effect of CNTF in A1s induction was significantly weakened after treating with miR-21a-5p mimic (Fig. 5A-D). Meanwhile, the effect of CNTF was enhanced with the transfection of miR-21a-5p inhibitor (Fig. 5E-H). Immunofluorescence staining showed that the effect of CNTF on decreasing C3 expression and increasing S100a10 expression in GFAP $^{+}$cells was enhanced after up-regulating miR-21a-5p (Fig. 5l-J) but weakened in miR-21a-5p knockdown group (Fig. 5K-L). In addition, miR-21a-5p overexpression slightly increased A1s markers without CNTF pre-treatment while down-regulating miR-21a-5p slightly decreased A1s markers and increased A2s markers. Those data illustrated that miR-21a-5p could repress the inhibitory effect of CNTF in A1s induction.

To determine whether miR-21a-5p inhibited the function of CNTF through targeting Cntfr a, we transfected Cntfr a siRNA into A1s with the treatment of miR-21a-5p knockdown. Firstly, we chose the siRNA contained the highest transfection efficiency through qRT-PCR and western blotting assay (Fig. 6A- 
B). As shown in Fig. 6C, cells transfected with Cntfr a siRNA showed a lower level of Cntfr a compared to those co-transfected with miR-21a-5p inhibitor. Significantly, qRT-PCR showed that the miR-21a-5p knockdown enhanced the inhibitory effect of CNTF on A1s induction, which was reversed by Cntfr a down-regulation (Fig. 6D-G). Immunofluorescence staining further confirmed that co-transfection with miR-21a-5p inhibitor and Cntfr a siRNA exhibited increased C3 expression and decreased S100a10 expression in $\mathrm{GFAP}^{+}$cells compared with cells treated with miR-21a-5p inhibitor (Fig. 6H-I). Meanwhile, we also detected the level of A1/A2 markers after cells transfected with Cntfr siRNA to clarify the function of Cntfr a whether affected by miR-21a-5p. Importantly, qRT-PCR and immunofluorescence staining showed higher A1s markers expression and lower A2s markers compared with the down-regulation of miR-21a-5p group.

In general, miR-21a-5p suppressed the function of CNTF on A1s induction through down-regulating Cntfr a.

\section{MiR-21 promoted A1s induction through down-regulating Cntfr a in vivo}

To confirm whether miR-21a-5p regulated the polarization of relative astrocytes in vivo, antagomir-21 was used to regulate the expression of miR-21a-5p in mice. Cntfr a siRNA was used to regulate the expression of Cntfr a in mice. As shown in Fig. 7A-B, the expression of miR-21a-5p in the antagomir-21 and antamir$21+$ Cntfr a siRNA group was decreased while the expression of Cntfr a was increased in antagomir-21 group, which effect can be abolished by Cntfr a knockdown. Significantly, qRT-PCR assay showed the decreased C3, Serping1, H2d1 expression and increased S100a10 after treatment with antagomir-21 which can be reversed by Cntfr a siRNA treatment (Fig. 7C-F), indicating that miR-21a-5p could promote the A1s induction via decreasing Cntfr a in vivo. In the early stage of $\mathrm{TSCl}$, the stimulation caused by ischemic injury might affect the polarization of reactive astrocytes in addition to neuroinflammation as there was higher $\mathrm{S} 100 \mathrm{a} 10$ expression in the $\mathrm{NC}$ TSCl group compared with the sham group (Fig. 7F). Subsequently, western blotting showed the phosphorylation of STAT3 was significantly increased by antagomir-21 treatment but decreased after treatment with Cntfr a siRNA after TSCl (Fig. 7G), which demonstrated that miR-21a-5p could affect STAT3 signal pathway through down-regulating Cntfr a after TSCl.

Furthermore, immunofluorescence staining showed the $\mathrm{C} 3$ expression in $\mathrm{GFAP}^{+}$cells was significantly down-regulated by antagomir-21 treatment, and recused by Cntfr a knockdown (Fig. 7H). Then the same trend was shown in immunohistochemistry assay (Fig. 7l).

Those data confirmed that miR-21a-5p promotes the induction of A1s through down-regulating Cntfr a after TSCl in vivo.

\section{Discussion}


Up to now, there is no effective treatment for axon regeneration, which is the main reason leading to poor prognosis of TSCI[1]. Promoting axonal regeneration and anti-inflammation are often used to treat TSCl in clinic[3]. Thus, it makes sense to regulate the alteration of reactive astrocytes from A1s to A2s, and then suppress inflammation for TSCl recovery.

In our study, we confirm for the first time that miR-21a-5p could promote the induction of A1 reactive astrocytes via CNTF/STAT3/Nkrf pathway after Traumatic Spinal Cord Injury (Fig. 8). Liddelow et al. reported that IL-1a, TNF- $a$ and C1q released by microglia might convert reactive astrocytes into a neurotoxic state in CNS disease[16]. Our study confirmed that A1s induction could be simulated by TSCI model in vivo. And astrocytes were transferred into A1s via treating with IL-1a, TNF-a and C1q in vitro. Based on this, the aim of our study was to explore key molecules regulated A1s polarization.

Our previous studies described that miR-21a-5p regulated glial scar formation[44] and inhibited the polarization of astrocytes to A2s in ISCI[18]. However, the effect of miR-21a-5p on neurotoxic reactive astrocytes (A1s) in TSCl has not been cleared. Interestingly, we found miR-21a-5p was up-regulated in A1s both in vivo and in vitro. Thus, we considered that miR-21a-5p may be an important factor for the polarization of relative astrocyte in $\mathrm{TSCl}$.

Concerning the potential mechanism of the miR-21a-5p-related reactive astrocytes polarization, gene chip assay and the bioinformatics analysis were used to explore targeting genes that could mediate the polarization of reactive astrocytes. Subsequently, we found four genes may exist binding site of miR-21a$5 p$. Upon further analysis, Cntfr a was confirmed to be targeted by miR-21a-5p. CNTFR a is a specific receptor of ciliary neurotrophic factor (CNTF), which was expressed in neuro, microglia, and astrocyte. Furthermore, it would activate the classical STAT3 signal pathway via binding with CNTF. Interestingly, when astrocytes were treated with CNTF after up-regulating miR-21a-5p, STAT3 signal pathway was weakened. Therefore, miR-21a-5p could down-regulate CNTF/STAT3 pathway and might be essential for regulating the induction of A1s. Nevertheless, it is still unclear how CNTF/STAT3 regulated the polarization of relative astrocytes.

Previous researches confirmed the positive effect of CNTF on reactive astrogliosis[23, 28-30] and M2 macrophages induction through activating classical STAT3 signal pathway[33]. Considering the important role of STAT3 in regulating the polarization of reactive astrocytes, we speculated that CNTF might regulate the polarization of reactive astrocytes from A1s to A2s by activating STAT3 signal pathway. In our study, we demonstrated that CNTF could down-regulate A1s markers and up-regulate A2s marker in vitro, which would be inhibited by repressing the activation of STAT3. But it is still unclear how transcription factor STAT3 affects A1s induction. NF-KB signal pathway is important for the polarization of $A 1$ reactive astrocytes $[15,35,36]$. Moreover, NF-KB repressing factor (Nkrf), a specific inhibitor of NF$\mathrm{KB}$ signaling pathway[48-50], might be related to the induction of M1 microglia and release the microglia-induced neuroinflammation[51]. However, the effect of Nkrf in reactive astrocytes is still unclear. Significantly, we found that CNTF could regulate the expression of Nkrf via promoting the 
transcription of STAT3, which was confirmed by qRT-PCR and ChIP assay. These data revealed the novel mechanism that CNTF regulated A1s polarization through STAT3/Nkrf pathway.

As miR-21a-5p was a key upstream molecule of CNTFR $a$, it was of great significance to verify whether miR-21a-5p could affect the function of CNTF/CNTFR $a$. Our data showed the effect of CNTF on A1s was strongly enhanced after down-regulating the expression of miR-21a-5p. Importantly, effects after downregulating miR-21a-5p were reduced when Cntfr a was knocked down. Down-regulating miR-21a-5p could also markedly regulate the polarization of reactive astrocytes which would reverse by Cntfr a knockdown in mice TSCl model. Interestingly, we also found that A1/A2 markers were slightly affected by miR-21a-5p without the treatment of CNTF in A1s. Further study would be performed to detect the mechanism. Thus, miR-21a-5p will be a key factor for targeted treatment of TSCl in the future.

Taken together, we confirmed that astrocyte-mediated neuroinflammation was regulated by miR-21a-5p. It is an innovative point of our research to explore the effect of miR-21a-5p on astrocytic

neuroinflammation after TSCl from another perspective. In addition, regulating miR-21a-5p could improve the environment for neuro regeneration. However, our study only referred to the expression of A1/A2 marker genes, but did not detect other astrocytic functions such as activity, migration and effects on neurons, which will be confirmed in our future study. And then, we demonstrated that CNTF inhibited A1s polarization through directly activating Nkrf. Is there any stimulating factor that directly promotes the alteration of reactive astrocytes to A2 induced by miR-21a-5p/CNTF/STAT3? We will further clarify it in future.

There is plenty of evidence to show that it is necessary for the recovery of TSCl to promote the alteration of reactive astrocytes from A1s to A2s. However, it remains to be detected whether other factors affect the alteration of A1/A2 reactive astrocytes. Are there only two types of reactive astrocytes? These problems will be solved through Single-cell sequencing, proteomics or other studies.

\section{Conclusions}

Collectively, our study confirmed that miR-21a-5p could promote the induction of A1s through Cntfr a/STAT3/Nkrf axis after TSCl, which may provide prospective sight for the reactive astrocytes alteration and promote the development of TSCl recovery.

\section{Abbreviations}

TSCI: Traumatic Spinal Cord Injury; miR: microRNA; CNTF: ciliary neurotrophic factor; CNTFR a: ciliary neurotrophic factor receptor $a ; C N S$ : central nervous system; A1s: neurotoxic reactive astrocyte; $A 2 \mathrm{~s}$ : neurotrophic reactive astrocyte; NC: negative control; siRNA: small interfering RNA; PBS: phosphatebuffered saline; IL-1a: interleukin 1a; TNF-a: tumor necrosis factor - a; C1q: complement component 1q; STAT3: signal transducer and activator of transcription-3; GFAP: glial fibrillary acidic protein; C3: complement component 3; Serping1: serpin family G member 1; $\mathrm{H} 2 \mathrm{~d} 1$ : histocompatibility 2, D region 
locus 1; S100a10: S100 calcium-binding protein A10; IL-1 $\beta$ : interleukin 1 $\beta$; iNOS: inducible nitric oxide synthase; GAPDH: glyceraldehyde 3-phosphate dehydrogenase; DAPI: 4',6-diamidino-2-phenylindole; NFкB: nuclear factor-kB; Nkrf: NF-kB repressing factor; qRT-PCR: quantitative real-time polymerase chain reaction; ELISA: Enzyme-linked immunosorbent assay; ChIP: chromatin immunoprecipitation.

\section{Declarations}

\section{Consent for Publication}

Not applicable

\section{Availability of Data and Materials}

The datasets used and/or analysed during the current study are available from the corresponding author on reasonable request.

\section{Ethics statement}

All operations are performed according to the animal care standards of the Chinese National Institute of Health and regulations of Shandong First Medical University Committee for the Care and Use of Laboratory Animals.

\section{Acknowledgements}

We thank the Research Center of Basic Medicine of Central Hospital Affiliated to Shandong First Medical University for the technical assistance. We are grateful to Dr. Ronghan Liu, Dr. Yanlin Su for their valuable discussions.

\section{Funding}

Grant support was provided by the National Natural Science Fund of China (Nos. 81401014, 81771346), the Taishan Scholar Program of Shandong Province(tsqn201812156), Spring City Leader Talent Support Plan, Rongxiang Regenerative Medicine Foundation of Shandong University (No. 2019SDRX-23).

\section{Authors' contributions}

Y.N.Z and B.N designed the study; Y.N.Z, T.M, J.C, Y.Z, J.K, X.L and G.Y performed the experiments; L.T, Z.J, H.D analyzed data; Y.N.Z wrote the manuscript; B.N helped to draft the manuscript; Authors read and approved the final manuscript.

\section{Conflict of interests}

The authors declare that there is no conflict of interests. 


\section{References}

1. O'Shea, T.M., J.E. Burda, and M.V. Sofroniew, Cell biology of spinal cord injury and repair. J Clin Invest, 2017. 127(9): 3259-70.

2. Sofroniew, M.V., Dissecting spinal cord regeneration. Nature, 2018. 557(7705): 343-50.

3. Zhou, P., et al., Cell Therapeutic Strategies for Spinal Cord Injury. Adv Wound Care (New Rochelle), 2019. 8(11): 585-605.

4. McDonald, J.W. and C. Sadowsky, Spinal-cord injury. Lancet, 2002. 359(9304): 417-25.

5. Ahuja, C.S., et al., Traumatic Spinal Cord Injury-Repair and Regeneration. Neurosurgery, 2017. 80(3s): S9-s22.

6. Tran, A.P., P.M. Warren, and J. Silver, The Biology of Regeneration Failure and Success After Spinal Cord Injury. Physiol Rev, 2018. 98(2): 881-917.

7. Sofroniew, M.V. and H.V. Vinters, Astrocytes: biology and pathology. Acta Neuropathol, 2010. 119(1): 7-35.

8. Karimi-Abdolrezaee, S. and R. Billakanti, Reactive astrogliosis after spinal cord injury-beneficial and detrimental effects. Mol Neurobiol, 2012. 46(2): 251-64.

9. Hara, M., et al., Interaction of reactive astrocytes with type I collagen induces astrocytic scar formation through the integrin-N-cadherin pathway after spinal cord injury. Nat Med, 2017. 23(7): 818-28.

10. Anderson, M.A., et al., Astrocyte scar formation aids central nervous system axon regeneration. Nature, 2016. 532(7598): 195-200.

11. Rothhammer, V. and F.J. Quintana, Control of autoimmune CNS inflammation by astrocytes. Semin Immunopathol, 2015. 37(6): 625-38.

12. Dokalis, N. and M. Prinz, Resolution of neuroinflammation: mechanisms and potential therapeutic option. Semin Immunopathol, 2019. 41(6): 699-709.

13. Sofroniew, M.V., Molecular dissection of reactive astrogliosis and glial scar formation. Trends Neurosci, 2009. 32(12): 638-47.

14. Zamanian, J.L., et al., Genomic analysis of reactive astrogliosis. J Neurosci, 2012. 32(18): 6391-410.

15. Liddelow, S.A. and B.A. Barres, Reactive Astrocytes: Production, Function, and Therapeutic Potential. Immunity, 2017. 46(6): 957-67.

16. Liddelow, S.A., et al., Neurotoxic reactive astrocytes are induced by activated microglia. Nature, 2017. 541(7638): 481-87.

17. Yun, S.P., et al., Block of A1 astrocyte conversion by microglia is neuroprotective in models of Parkinson's disease. Nat Med, 2018. 24(7): 931-38.

18. Su, Y., et al., Silencing miR-21 induces polarization of astrocytes to the A2 phenotype and improves the formation of synapses by targeting glypican 6 via the signal transducer and activator of transcription-3 pathway after acute ischemic spinal cord injury. Faseb j, 2019. 33(10): 10859-71. 
19. Vismara, I., et al., Selective Modulation of A1 Astrocytes by Drug-Loaded Nano-Structured Gel in Spinal Cord Injury. ACS Nano, 2020. 14(1): 360-71.

20. Fujita, A., et al., Connexin 30 deficiency attenuates $A 2$ astrocyte responses and induces severe neurodegeneration in a 1-methyl-4-phenyl-1,2,3,6-tetrahydropyridine hydrochloride Parkinson's disease animal model. J Neuroinflammation, 2018. 15(1): 227.

21. Neal, M., et al., Prokineticin-2 promotes chemotaxis and alternative $A 2$ reactivity of astrocytes. Glia, 2018. 66(10): 2137-57.

22. Wen, R., et al., CNTF and retina. Prog Retin Eye Res, 2012. 31(2): 136-51.

23. Pasquin, S., M. Sharma, and J.F. Gauchat, Ciliary neurotrophic factor (CNTF): New facets of an old molecule for treating neurodegenerative and metabolic syndrome pathologies. Cytokine Growth Factor Rev, 2015. 26(5): 507-15.

24. Dallner, C., et al., CNTF and CNTF receptor alpha are constitutively expressed by astrocytes in the mouse brain. Glia, 2002. 37(4): 374-8.

25. Leibinger, M., et al., Neuronal STAT3 activation is essential for CNTF- and inflammatory stimulationinduced CNS axon regeneration. Cell Death Dis, 2013. 4(9): e805.

26. Luo, X., et al., Enhanced Transcriptional Activity and Mitochondrial Localization of STAT3 Co-induce Axon Regrowth in the Adult Central Nervous System. Cell Rep, 2016. 15(2): 398-410.

27. Anderson, M.A., et al., Required growth facilitators propel axon regeneration across complete spinal cord injury. Nature, 2018. 561(7723): 396-400.

28. Hudgins, S.N. and S.W. Levison, Ciliary neurotrophic factor stimulates astroglial hypertrophy in vivo and in vitro. Exp Neurol, 1998. 150(2): 171-82.

29. Albrecht, P.J., et al., Ciliary neurotrophic factor activates spinal cord astrocytes, stimulating their production and release of fibroblast growth factor-2, to increase motor neuron survival. Exp Neurol, 2002. 173(1): 46-62.

30. Herrmann, J.E., et al., STAT3 is a critical regulator of astrogliosis and scar formation after spinal cord injury. J Neurosci, 2008. 28(28): 7231-43.

31. Krady, J.K., et al., Ciliary neurotrophic factor and interleukin-6 differentially activate microglia. J Neurosci Res, 2008. 86(7): 1538-47.

32. Baek, J.Y., et al., Inhibition of Microglia-Derived Oxidative Stress by Ciliary Neurotrophic Factor Protects Dopamine Neurons In Vivo from MPPQ Neurotoxicity. Int J Mol Sci, 2018. 19(11) :3543.

33. Blanco, R.E., et al., Application of CNTF or FGF-2 increases the number of M2-like macrophages after optic nerve injury in adult Rana pipiens. PLoS One, 2019. 14(5): e0209733.

34. Gensel, J.C. and B. Zhang, Macrophage activation and its role in repair and pathology after spinal cord injury. Brain Res, 2015. 1619: 1-11.

35. Wang, L., et al., Mesenchymal Stem Cell-Derived Exosomes Reduce A1 Astrocytes via Downregulation of Phosphorylated NFKB P65 Subunit in Spinal Cord Injury. Cell Physiol Biochem, 2018. 50(4): 153559. 
36. Xu, X., et al., MFG-E8 reverses microglial-induced neurotoxic astrocyte (A1) via NF-KB and PI3K-Akt pathways. J Cell Physiol, 2018. 234(1): 904-14.

37. Yamashita, T., et al., Blockade of interleukin-6 signaling aggravates ischemic cerebral damage in mice: possible involvement of Stat3 activation in the protection of neurons. J Neurochem, 2005. 94(2): 459-68.

38. Wanner, I.B., et al., Glial scar borders are formed by newly proliferated, elongated astrocytes that interact to corral inflammatory and fibrotic cells via STAT3-dependent mechanisms after spinal cord injury. J Neurosci, 2013. 33(31): 12870-86.

39. Renault-Mihara, F. and H. Okano, STAT3-regulated RhoA drives reactive astrocyte dynamics. Cell Cycle, 2017. 16(21): 1995-6.

40. Lu, T.X. and M.E. Rothenberg, MicroRNA. J Allergy Clin Immunol, 2018. 141(4): p. 1202-7.

41. Mohr, A.M. and J.L. Mott, Overview of microRNA biology. Semin Liver Dis, 2015. 35(1): 3-11.

42. Nieto-Diaz, M., et al., MicroRNA dysregulation in spinal cord injury: causes, consequences and therapeutics. Front Cell Neurosci, 2014. 8: 53.

43. Ning, B., et al., microRNAs in spinal cord injury: potential roles and therapeutic implications. Int J Biol Sci, 2014. 10(9): 997-1006.

44. Liu, R., et al., microRNA-21 regulates astrocytic reaction post-acute phase of spinal cord injury through modulating TGF- $\beta$ signaling. Aging (Albany NY), 2018. 10(6): 1474-88.

45. Wang, W., et al., MicroRNA-21-5p mediates TGF- $\beta$-regulated fibrogenic activation of spinal fibroblasts and the formation of fibrotic scars after spinal cord injury. Int J Biol Sci, 2018. 14(2): 178-88.

46. Wang, W., et al., MicroRNA-21a-5p promotes fibrosis in spinal fibroblasts after mechanical trauma. Exp Cell Res, 2018. 370(1): 24-30.

47. Xie, W., et al., Knockdown of MicroRNA-21 Promotes Neurological Recovery After Acute Spinal Cord Injury. Neurochem Res, 2018. 43(8): 1641-9.

48. Nourbakhsh, M., et al., NRF, a nuclear inhibitor of NF-kappaB proteins silencing interferon-beta promoter. Eur Cytokine Netw, 2000. 11(3): 500-1.

49. Nourbakhsh, M. and H. Hauser, Constitutive silencing of IFN-beta promoter is mediated by NRF (NFkappaB-repressing factor), a nuclear inhibitor of NF-kappaB. Embo j, 1999. 18(22): 6415-25.

50. Lee, K.Y., et al., Reduced nuclear factor-KB repressing factor: a link toward systemic inflammation in COPD. Eur Respir J, 2012. 40(4): 863-73.

51. Hazra, B., et al., miR-301a Regulates Inflammatory Response to Japanese Encephalitis Virus Infection via Suppression of NKRF Activity. J Immunol, 2019. 203(8): 2222-38.

\section{Tables}

Table. 1 Sequence of primer pairs used in the study 


\begin{tabular}{|c|c|c|}
\hline \multirow[b]{2}{*}{ Gene } & \multicolumn{2}{|l|}{ Primer sequence, $5^{\prime}-3^{\prime}$} \\
\hline & Forward & Reverse \\
\hline mmu-miR-21a-5p & CCTAGCTTATCAGACTGATGTTGA & \\
\hline U6 & GGAACGATACAGAGAAGATTAGC & TGGAACGCTTCACGAATTTGCG \\
\hline Cntfra & TCCCAGGAAGACTTTGGTCTGG & CTGTGGACTGTGTTTCTGCGTGT \\
\hline C3 & GCAGACCTTAGCGACCAAGT & CCGCAATGACTGTTGGTGTC \\
\hline Serping1 & TACGATCTCAACCTGTGCGG & AGTTCCAGCACTGTCTCGTG \\
\hline $\mathrm{H} 2 \mathrm{~d} 1$ & GCATTACAAGGCCTACCTGGA & CAGCACCTCAGGGTGACTTC \\
\hline S100a10 & TACGTTTCACAGGTTTGCAGG & TCCCGTTCCATGAGCACTCT \\
\hline iNOS & CCTGCTTTGTGCGAAGTGTC & CCCAAACACCAAGCTCATGC \\
\hline$\|-1 \beta$ & TCCAGGATGAGGACATGAGCAC & GAACGTCACACACCAGCAGGTTA \\
\hline Nkrf & CACTCAGGCTCTTCACCCAA & GCGGAGACCTGTCATCCTTT \\
\hline Epha4 & GCAATCCCAACAGCCTGAAGA & CAGCCAGTCGCCCACTGATA \\
\hline Pitx2 & AACCTTACGGAAGCCCGAGTC & CCCAAAGCCATTCTTGCACA \\
\hline Abad2 & TGACCAAGACCTAGAACGCATCC & TTTCCAGTCCATGACTGCATCC \\
\hline GAPDH & TGTCTCCTGCGACTTCAACA & GGTGGTCCAGGGTTTCTTACT \\
\hline
\end{tabular}

\section{Figures}


A

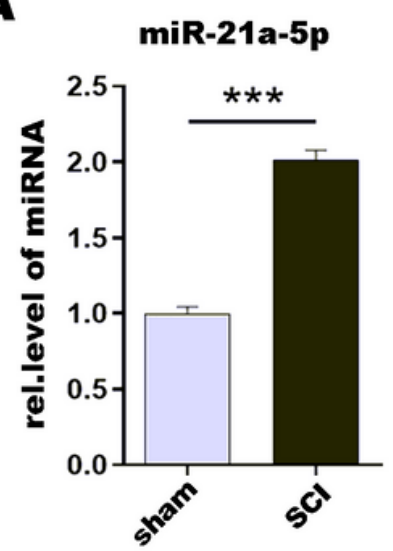

C

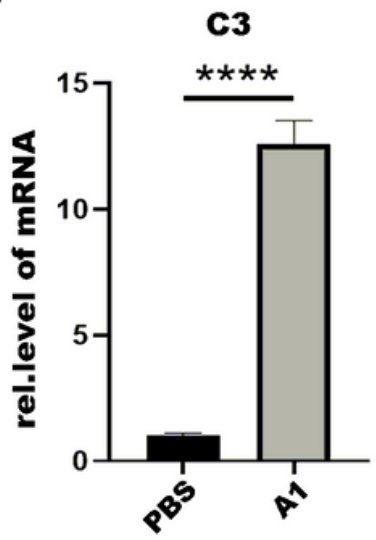

D

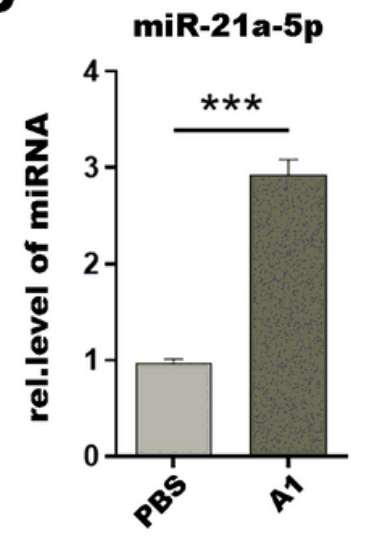

$\mathbf{B}$
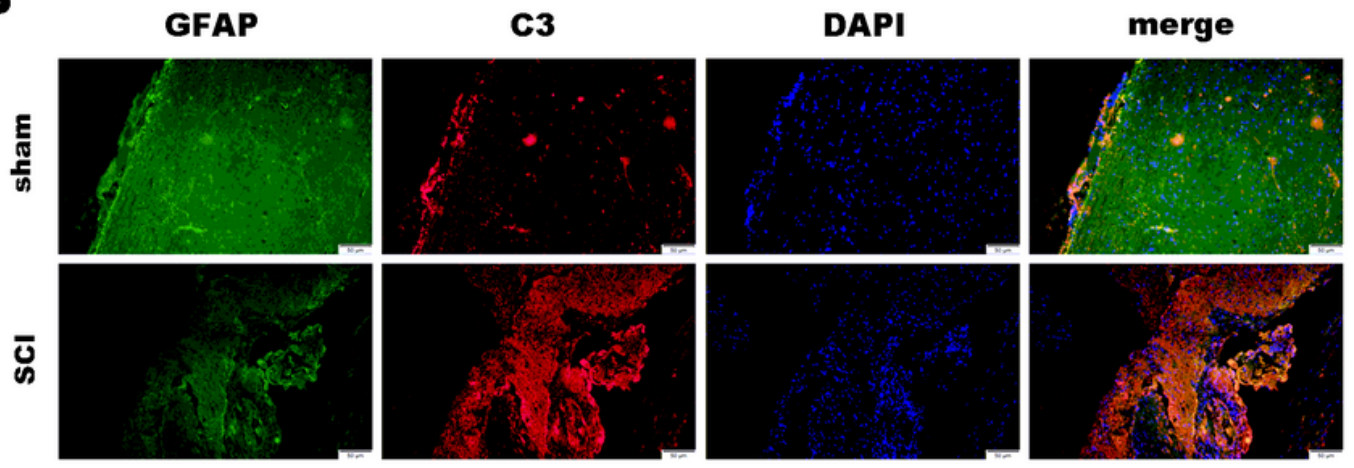

ত্র

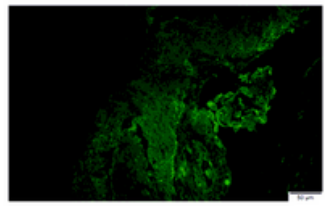

Serping1

H2d1

S100a10

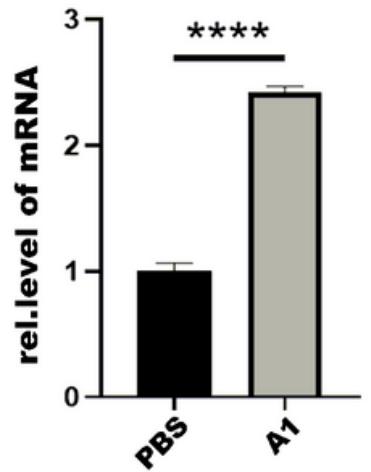

$\mathbf{E}$

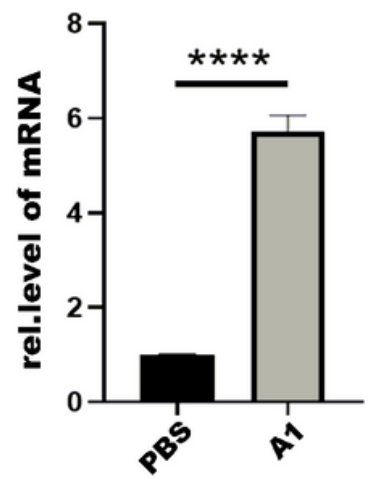

$\mathbf{F}$

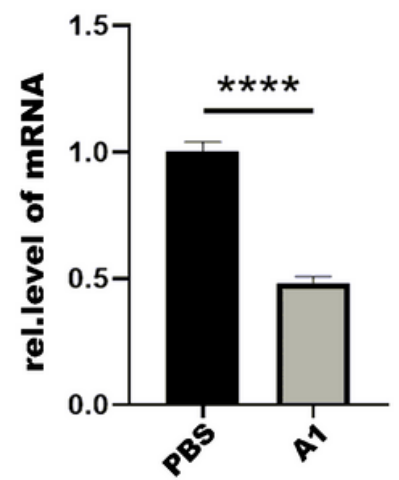

$F$


Figure 1

Neurotoxic astrocyte (A1) appeared 3 days after Traumatic Spinal Cord Injury, with the increase of miR21a-5p and the decrease of Cntfr a. (A). The miR-21a-5p expression in 3d post-TSCl was described by qRT-PCR. (B). The expressions of GFAP (green) and C3 (red) in sham operation and 3d post-TSCl group

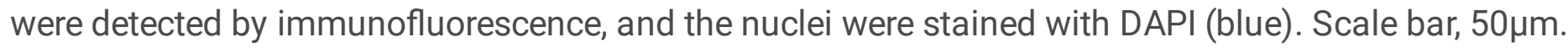
The transformation of naive astrocytes into A1s was induced by IL-1a, TNF-a, and C1q. (C). qRT-PCR was used to detect the mRNA expression of C3, Serping1, H2D1, S100a10, GAPDH was used for normalization. (D). qRT-PCR was used to detect the expression of miR-21a-5p, U6 was used for normalization. (E). Heat map of mRNA that significantly changed in 3d post-TSCl group and bioinformatics analysis for choosing targeting genes of miR-21a-5p. (F). qRT-PCR was used to detect the 

results were analyzed by GraphPad and SPSS. The data are expressed in terms of mean $\pm S D, n=3$. ${ }^{*}$ $<0.05, * * P<0.01, * * * P<0.001, * * * * p<0.0001$.

A

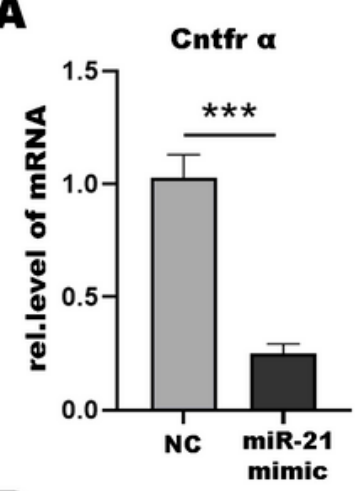

B

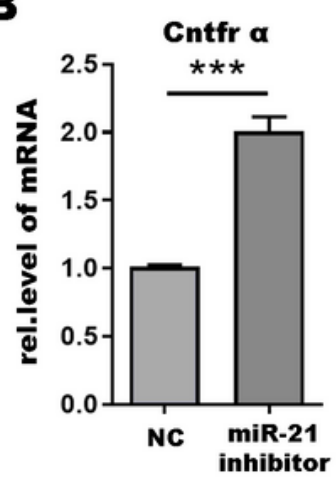

C miR-21a-5p: AGUUGUAGUCAGAC U'U'UUU'C'Ǵ' U

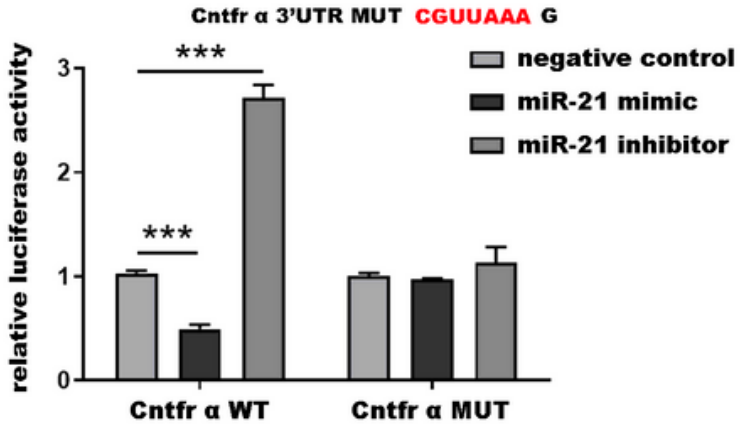

G
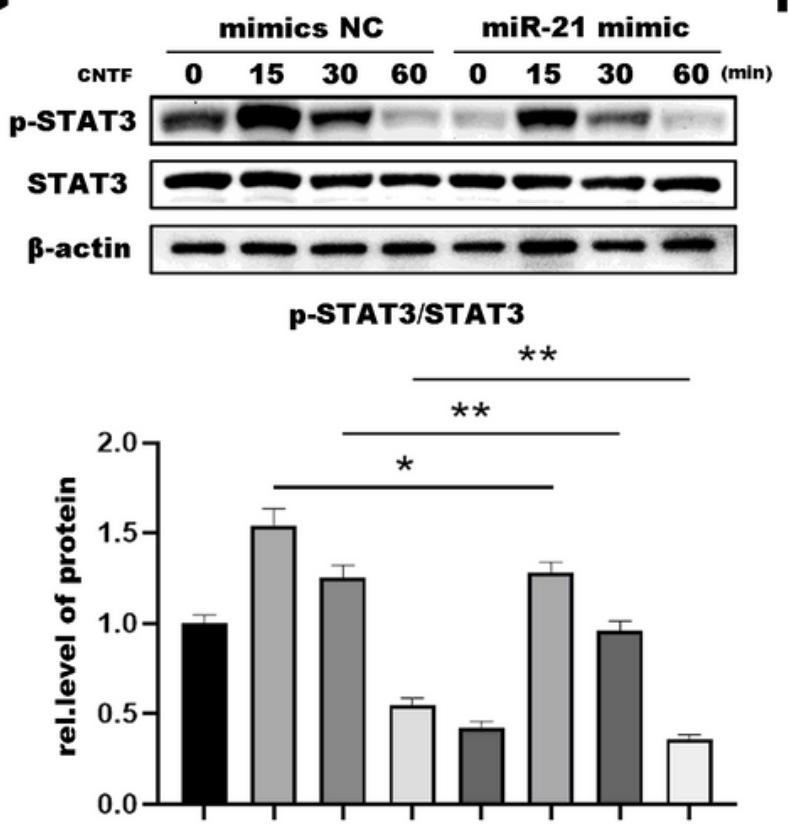



E

F miR-21 pulldown

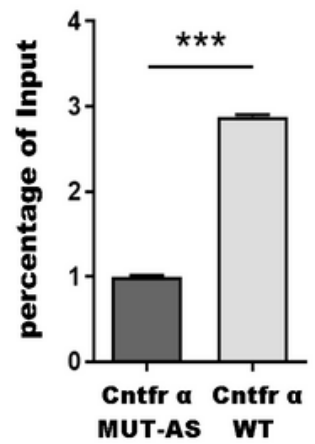

H

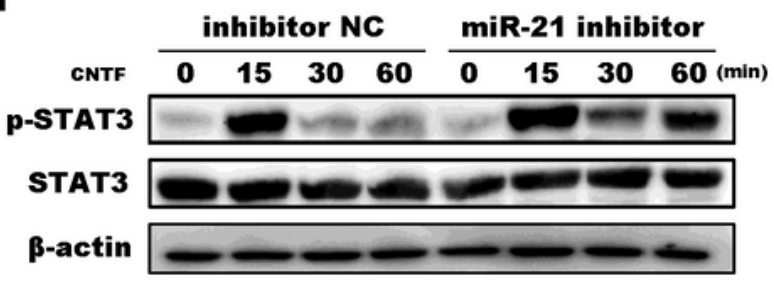

p-STAT3/STAT3



Figure 2

miR-21a-5p downregulated the expression of Cntfr a by targeting 3'UTR. miR-21a-5p mimic, inhibitor, and negative control were transfected into astrocytes. (A, B). qRT-PCR was used to detect the mRNA level of 
Cntfr a, normalized by GAPDH. (C). Western blotting was used to detect the protein level of CNTFR a, normalized by $\beta$-actin. (D). Prediction of targeting sequence between miR-21a-5p and Cntfr; Dual-

luciferase reporter assays were performed to determine the targeting sequence of miR-21a-5p and Cntfr a. (E). Astrocyte lysate used for RNA pulldown assay, then tested the expression of miR-21a-5p with qRTPCR, relative levels of miR-21a-5p were normalized by Input. $(F)$. miR-21 inhibitor was used to downregulate the expression of miR-21a-5p in astrocytes, and then naive astrocytes were induced into $A 1 s$. The expression of Cntfr a was detected by qRT-PCR and normalized by GAPDH. (G, H). Astrocytes were transfected with miR-21a-5p mimic, inhibitor, and negative control, then treated with CNTF for 0, 15 30, 60 minutes, western blotting was used to detect the expression of p-STAT3 and STAT3, $\beta$-actin. The results were analyzed by Image J, GraphPad, and SPSS. The data are expressed in terms of mean $\pm S D, n=3$. *P

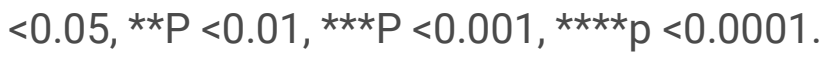



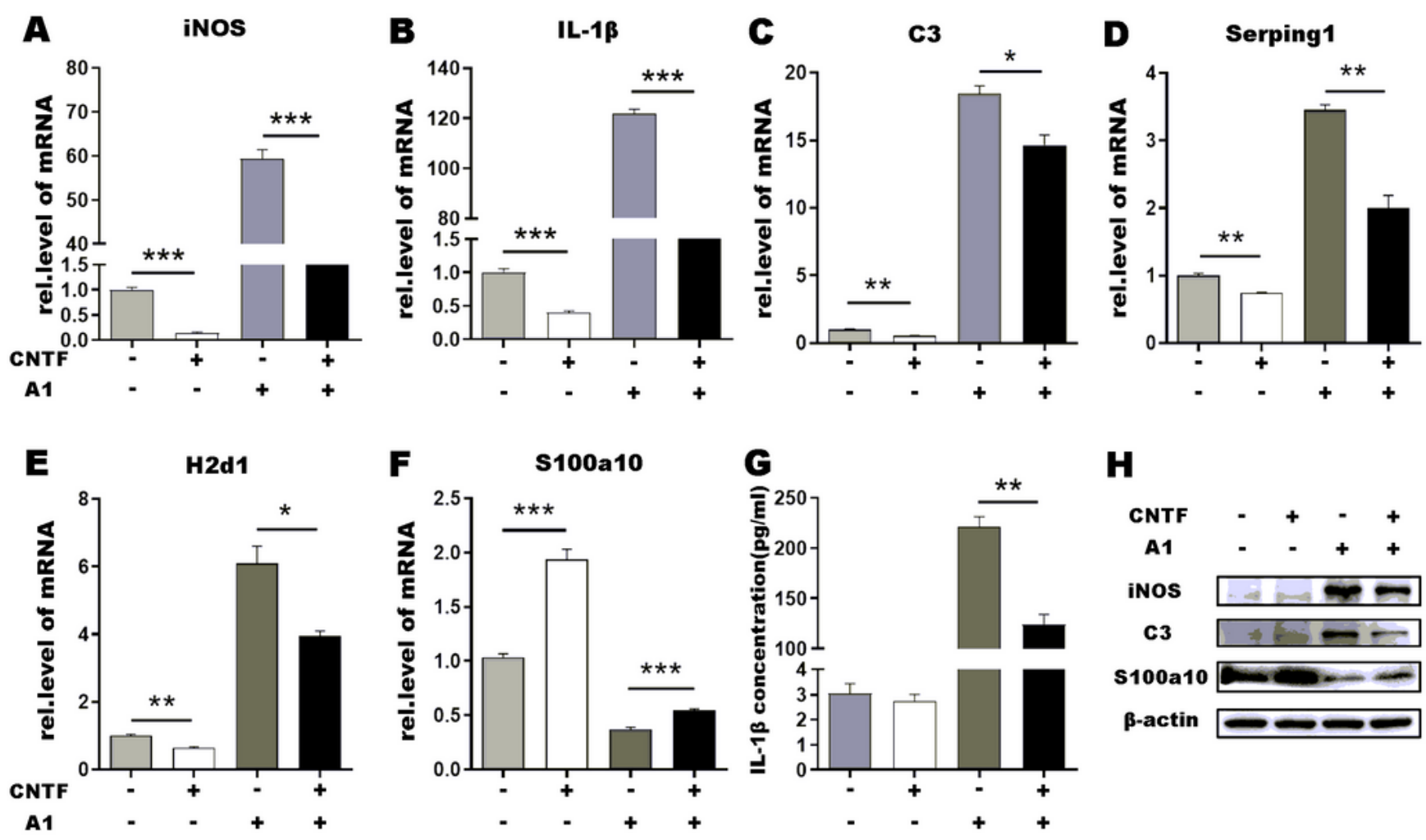

H
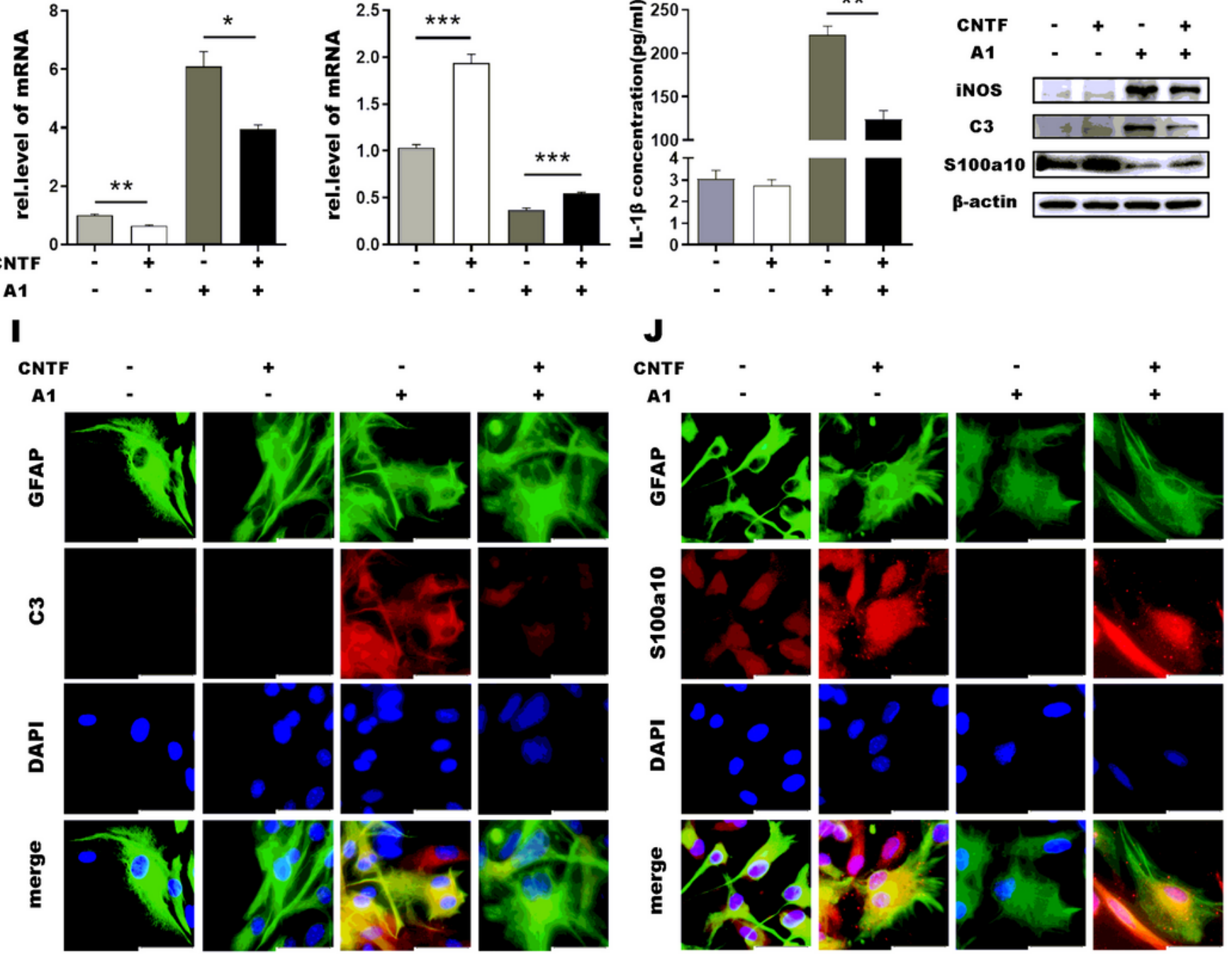

Figure 3

CNTF down-regulated the polarization of neurotoxic astrocytes (A1). After pre-treating with CNTF, native astrocytes were induced into $A 1$ reactive astrocytes by IL-1, TNF, and C1q. PBS was used as an untreated group. (A-F) The mRNA expressions of iNOS, IL-1 $\beta$, C3, Serping1, H2D1, and S100a10 were detected by qRT-PCR, and GAPDH was used for normalization. (G) The concentration of IL-1 $\beta$ released by astrocytes was detected by ELISA. The results were analyzed by GraphPad and SPSS. The data were expressed in 
terms of mean $\pm S D, n=3 .{ }^{*}<<0.05$, ${ }^{\star *} P<0.01$, ${ }^{\star \star *} P<0.001$, ${ }^{\star \star \star \star} p<0.0001$. (H) iNOS, C3, S100a10, and $\beta$ actin were detected by western blotting, $n=3$. (I-J) Immunofluorescence were used to detect GFAP (green; IJ), C3 (red; I), S100a10 (red; J) and DAPI (blue; nuclei). Scale bar, 20 m. N=3.

A

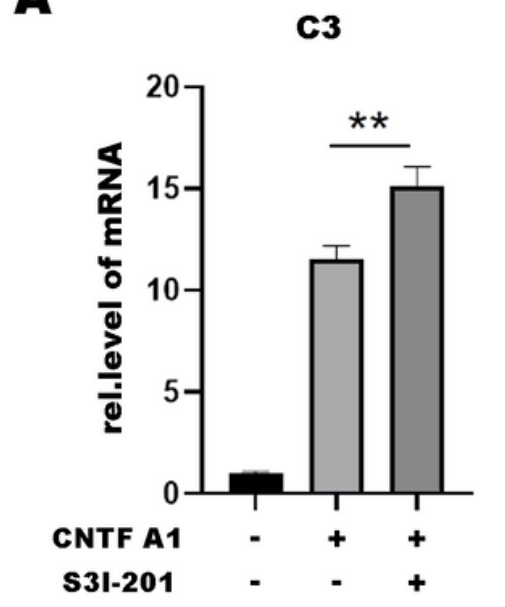

$\mathbf{E}$

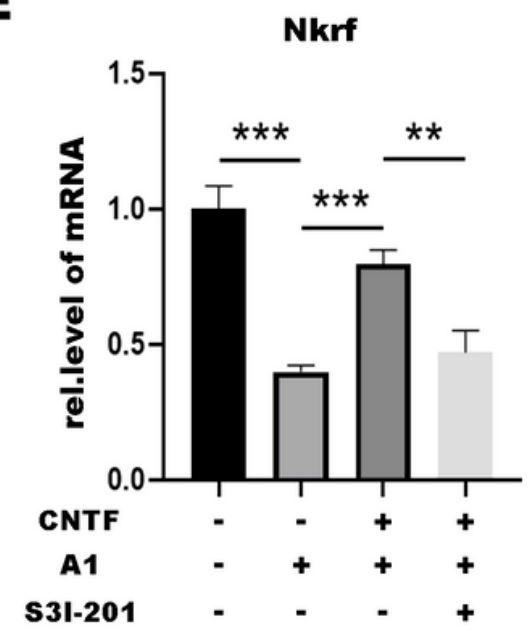

$\mathbf{B}$



$\mathbf{F}$
C

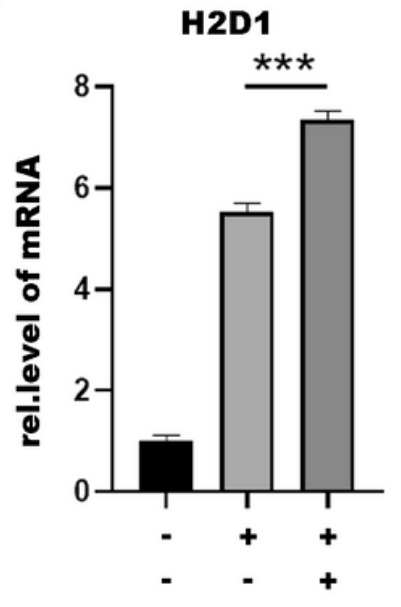

D

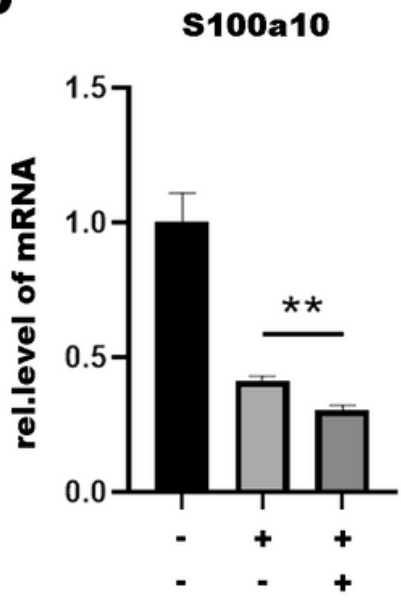

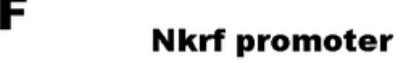

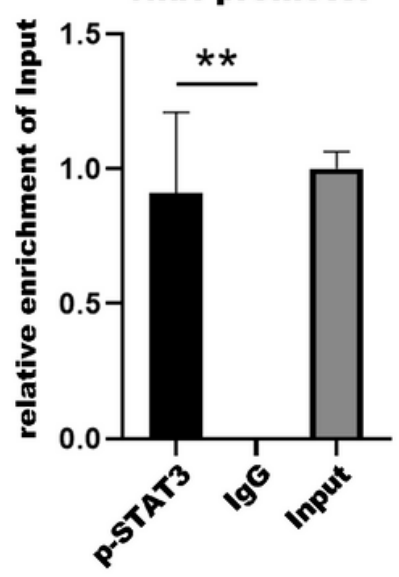

Nkrf promoter

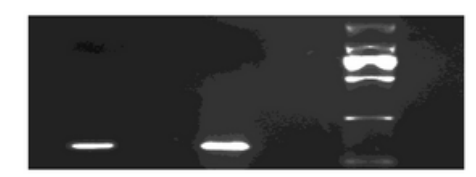

\section{Figure 4}

CNTF downregulated A1s by promoting Nkrf through STAT3 signaling pathway. (A-D) Astrocytes were treated with S3I-201 for 1 hour and DMSO was used as untreated. After pre-treatment with CNTF, astrocytes were induced into A1s. The expression of C3, Serping1, H2D1, and S100a10 mRNA were detected by qRT-PCR. (E) The expression of Nkrf mRNA was detected by qRT-PCR, normalized by GAPDH. (F) The targeted binding between p-STAT3 and Nkrf promoters was detected by ChIP assay, and Input was used for normalization. The results were analyzed by GraphPad and SPSS. The data were expressed in terms of mean $\pm S D, n=3 .{ }^{*} P<0.05,{ }^{\star} \mathrm{P}<0.01$, ${ }^{\star \star *} \mathrm{P}<0.001$, ${ }^{\star \star \star \star} \mathrm{p}<0.0001$. 


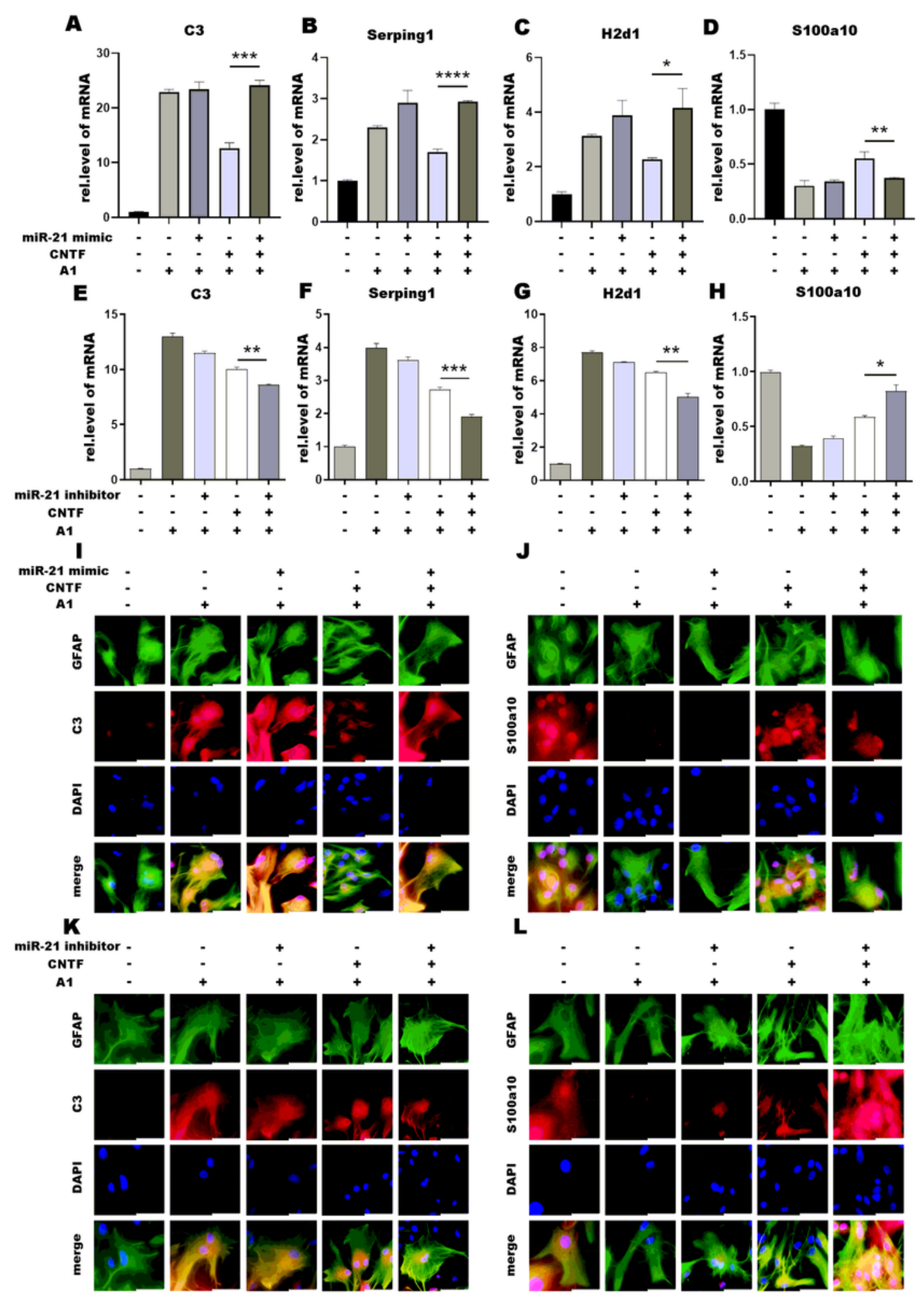

Figure 5

miR-21a-5p downregulated the inhibitory effect of CNTF on neurotoxic astrocytes (A1). miR-21 mimic, inhibitor, and negative control were transfected into astrocytes. After pre-treating with CNTF, native astrocytes were induced into A1s. (A-H) qRT-PCR was used to detect the expression of C3, Serping1, H2D1, and S100a10 mRNA, normalized by GAPDH. The results were analyzed by GraphPad and SPSS. The data were expressed in terms of mean $\pm S D, n=3$. ${ }^{*} P<0.05$, ${ }^{* \star} P<0.01$, ${ }^{* \star *} P<0.001$, ${ }^{* \star * \star} p<0.0001$. (I-L) 
Astrocytes were detected by immunofluorescence staining with GFAP (green; I-L), C3 (red; I, K), S100a10 (red; J, L), and DAPI (blue; nuclei). Scale bar, $20 \mu \mathrm{m} . \mathrm{N}=3$.

A

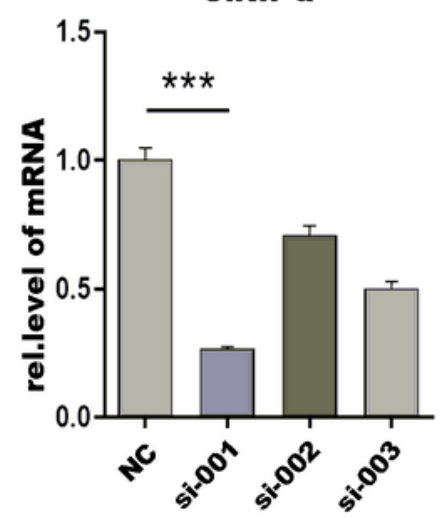

B

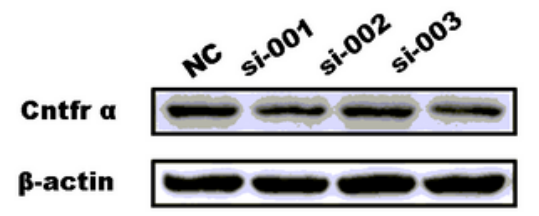

C

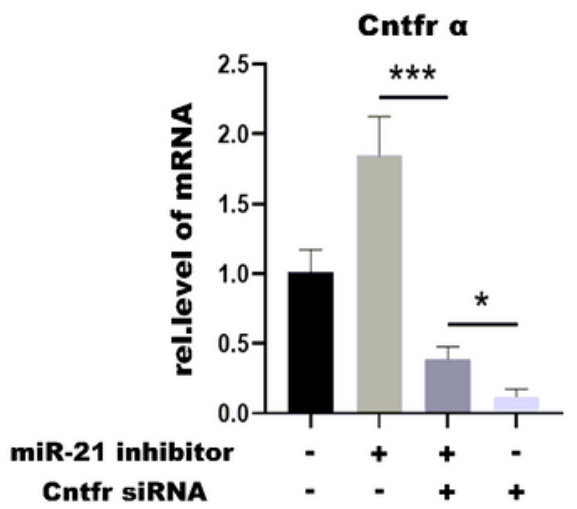

$\mathbf{F}$
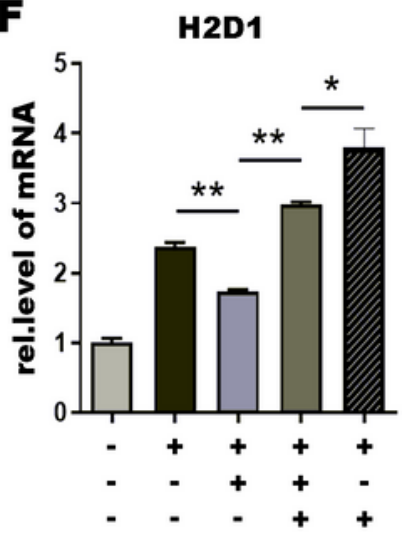

G
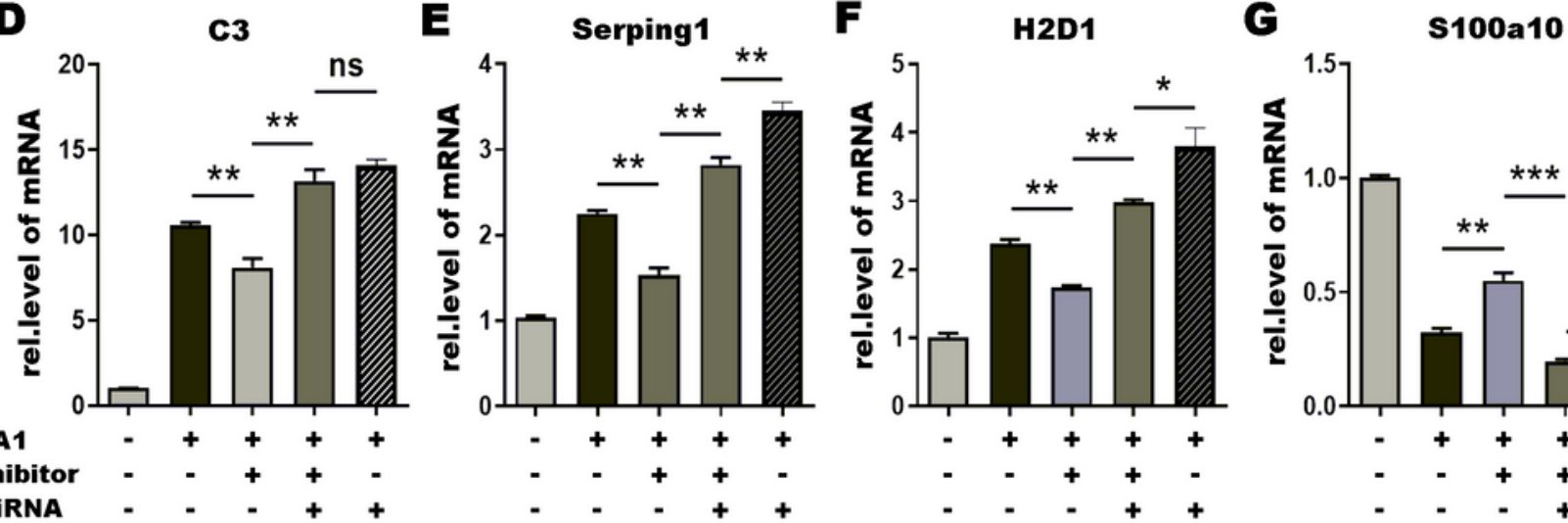

E Serping1

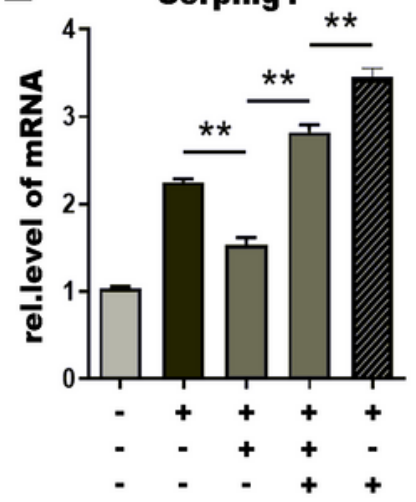

I
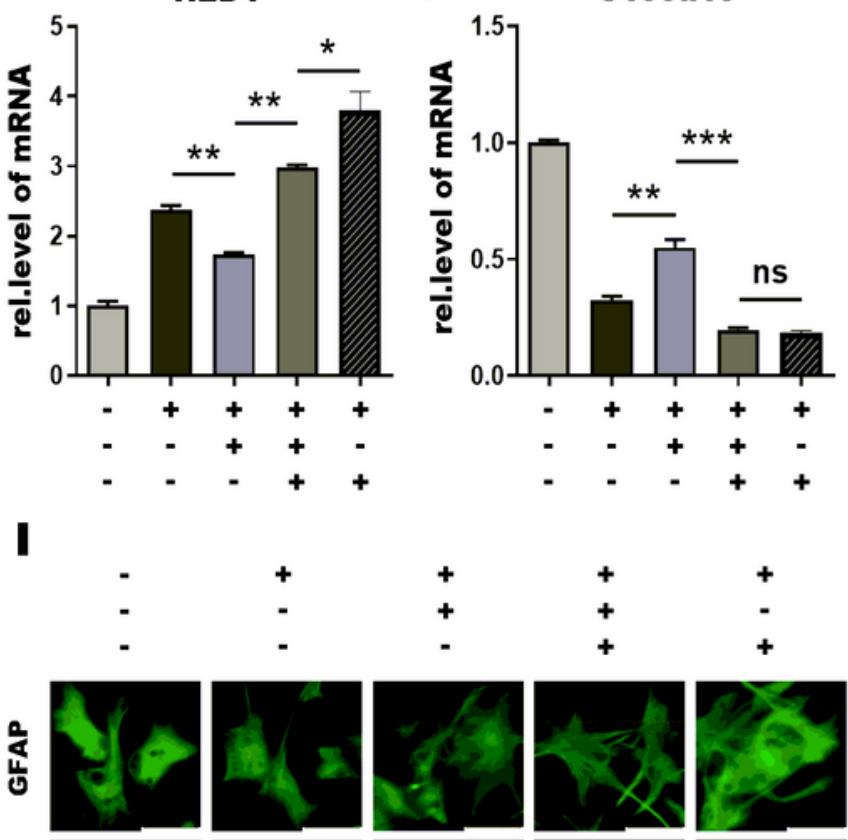

CNTF A1
miR-21 inhibitor
Cntfr a siRNA


Figure 6

miR-21a-5p inhibited the effect of CNTF by inhibiting the expression of Cntfr a. 3 kinds of Cntfr a siRNA were transfected into astrocytes. The expression of Cntfr a mRNA was detected by qRT-PCR (A). The expression of CNTFR a protein was detected by western blotting (B). (C) miR-21 inhibitor, Cntfr a siRNA, 
and negative controls were transfected into astrocytes, and the expression of Cntfr a mRNA was detected by qRT-PCR, normalized by GAPDH. (D-G) miR-21 inhibitor, Cntfr a siRNA, and negative controls were transfected into astrocytes. After pre-treating with CNTF, astrocytes were induced into A1s. The expression of C3, Serping1, H2D1, and S100a10 mRNA were detected by qRT-PCR and normalized by GAPDH. The results were analyzed by GraphPad and SPSS. The data were expressed in terms of mean $\pm S D, n=3 .{ }^{*} P<0.05,{ }^{*} \mathrm{P}<0.01,{ }^{\star * \star} \mathrm{P}<0.001$, ${ }^{\star \star \star \star} \mathrm{p}<0.0001$. $(\mathrm{H}-\mathrm{I})$ immunofluorescence was used to detect GFAP (green; H-I), C3 (red; H), S100a10 (red; I) and DAPI (blue; nuclei), n=3.

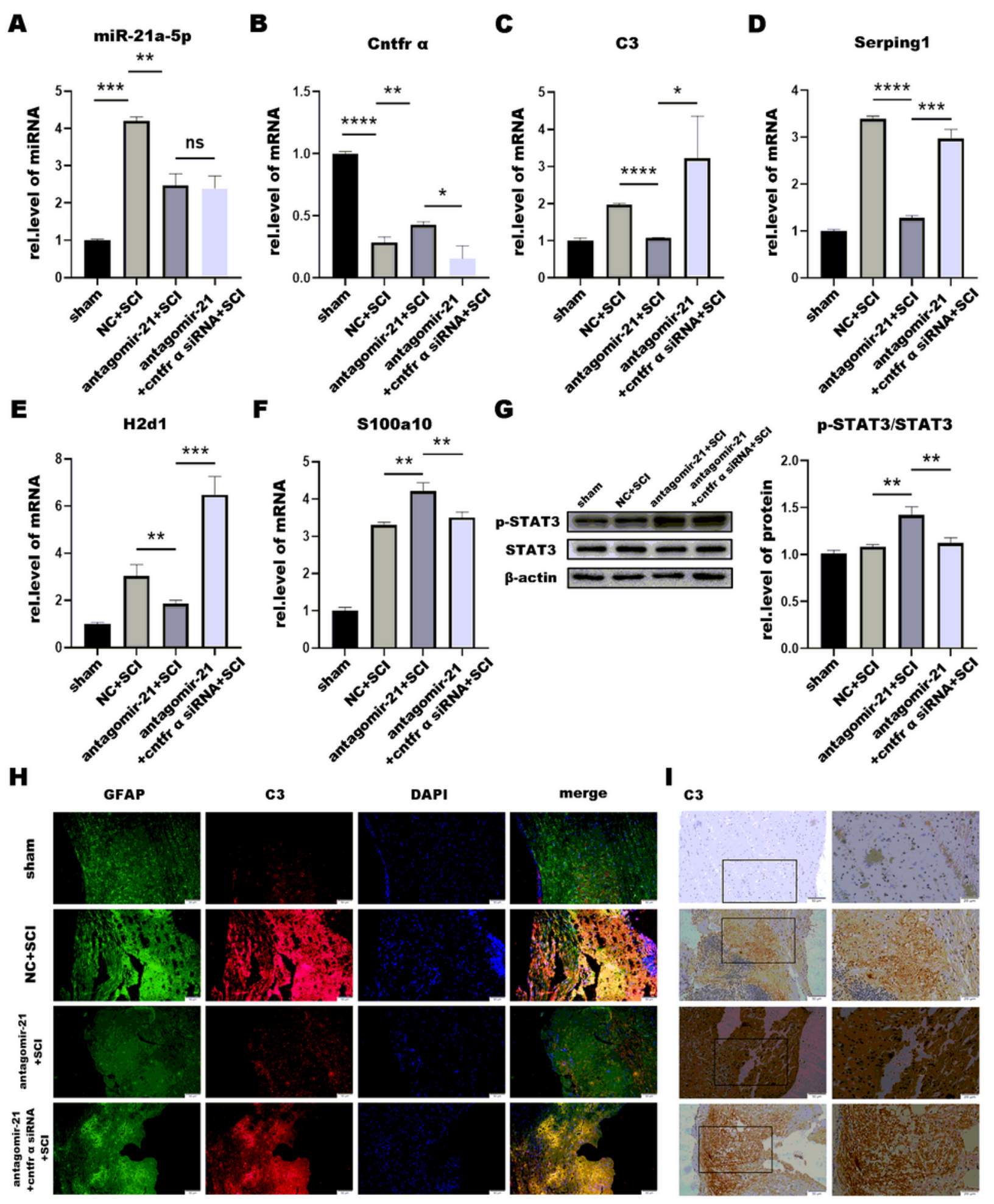




\section{Figure 7}

miR-21 promoted the polarization of neurotoxic astrocytes (A1) by inhibiting the expression of Cntfr a in vivo. Mice were divided into 4 groups: sham, $\mathrm{NC}+\mathrm{TSCl}$, antagomir-21+TSCl, antagomir-21+Cntfr a siRNA+TSCl. (A) miR-21 expression was detected by qRT-PCR and normalized by U6. (B-F) The expressions of Cntfr a, C3, Serping1, H2D1, and S100a10 mRNA were detected by qRT-PCR and normalized by GAPDH. The results were analyzed by GraphPad and SPSS. The data were expressed in terms of mean $\pm S D, n=3 .{ }^{*} P<0.05,{ }^{*} P<0.01$, ${ }^{* \star *} P<0.001$, ${ }^{\star \star *} \mathrm{p}<0.0001$. (G) Western blotting was used to detect the expression of p-STAT3, STAT3, and $\beta$-actin protein. The results were analyzed by Image $\mathrm{J}$, GraphPad, and SPSS software. The data were expressed in terms of mean $\pm S D, n=3 .{ }^{*} P<0.05,{ }^{*} P<0.01$, $\star \star \star * P<0.001, * \star \star \star p<0.0001$. (H) immunofluorescence was used to detect GFAP (green), C3 (red), and DAPI (blue; nuclei). Scale bar, $50 \mu \mathrm{m}$. (I) immunohistochemistry was used to detect C3. Scale bar, low magnification: $50 \mu \mathrm{m}$; high magnification: $20 \mu \mathrm{m} . \mathrm{N}=3$.

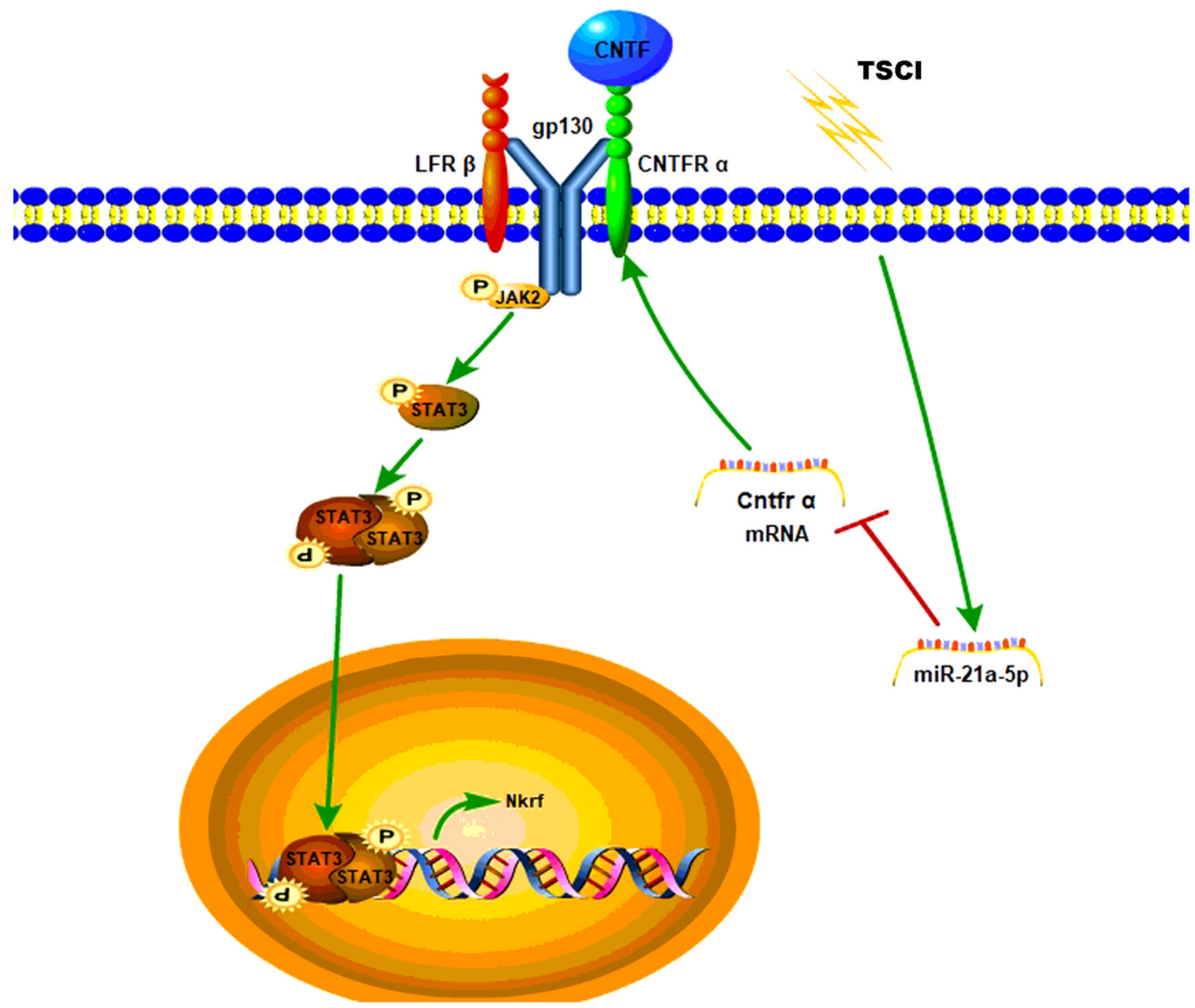

Figure 8 
The pathway of miR-21a-5p regulates the polarization of relative astrocytes through Cntfr/STAT3/Nkrf axis after TSCl.

\section{Supplementary Files}

This is a list of supplementary files associated with this preprint. Click to download.

- SupplementaryTable.docx

- zynsfig1.tif

- zynsfig2.tif 